\title{
Quality Factor Study for Cone-metal Shelled Structure
}

\author{
Mohammed Nadhim Abbas \\ Electrical Engineering Department, \\ College of Engineering, \\ University of Baghdad, Baghdad, \\ Iraq
}

\begin{abstract}
Pure plasmonic mode and hybrid plasmonic mode have been demonstrated. The cone-metal shelled structure is proposed. The two modes have been examined, pure plasmonic mode and hybrid plasmonic mode. At room temperature, the quality factor of the two modes has been compared and we found that the quality factor of hybrid plasmonic mode is 775 when the side angle of the cone structure ranged $\left(60^{\circ}-70^{\circ}\right)$ whereas the quality factor for pure plasmonic mode is 310 when the side angle of the cone structure ranged $\left(60^{\circ}-70^{\circ}\right)$. Also, we examined a quality factor of two modes at different temperature and we got an ultra high quality factor for pure plasmonic mode about $1.4 \mathrm{M}$ at $0 \mathrm{~K}$ whereas the quality factor for hybrid plasmonic mode is about 70,000 at $0 \mathrm{~K}$.
\end{abstract}

\section{General Terms}

Plasmonic, hybrid plasmonic mode, nanoparticles.

\section{Keywords}

Plasmonics, Surface plasmon polration, Quality factor

\section{INTRODUCTION}

In recent years, a substantial amount of research has been conducted in minimizing optical cavities to achieve nanoscale lasers used for chip-scale photonic integrated circuits. The metal-cladding semiconductor laser is one of the most promising schemes for breaking the optical diffraction limit because of surface plasmon (SP) effects [1,2]. Semiconductor metal lasers with different cavities[3-6] , including nanodisks, nanowires, nanorings, and other structures, have been demonstrated. Plasmonic resonant cavities represent an application that could exploit this field compression to create ultrasmall-mode-volume devices. Several plasmonic resonators with different geometrical designs have been reported, such as periodic optical Bragg mirrors [7 -12], annular nanoresonators [13 ], nanowaveguide-type cavity [14 ] and sandwiched Fabry-Perot (FP)-type nanocavity [15 ], but their quality factors are generally low, smaller than 100, which is far away from the theoretical value limited by the intrinsic loss of the metal at room temperature. In this paper, it has been investigated cone-metal shelled structure. Two kinds of modes have been focused in this structure, pure plasmonic mode and hybrid plasmonic mode. The quality factors of the two modes at room temperature and low temperature are compared.

\section{PLASMONIC RESONATOR}

\subsection{Study Model}

To understand the optical properties in the cone-metal shelled structure, it is used the three-dimensional (3D) finite-element method (FEM) was applied to perform the simulation of cone cavity covered by metallic shell as depicted in figure 1 (a), which consists of dielectric cone with height (h) of $2.4 \mathrm{~nm}$ and base diameter of 3 um. Cone structure is coated with metal layer $(\sim 100 \mathrm{~nm}$ thick $)$. The angle $\Phi$ represents the angle between the sidewall and the base of the cone structure as shown in figure 1(b). The material of cone structure is $\mathrm{InP}$ and the metallic layer is Ag. The frequency-dependent complex dielectric constants of InP and $\mathrm{Ag}$ are taken from [16 ].

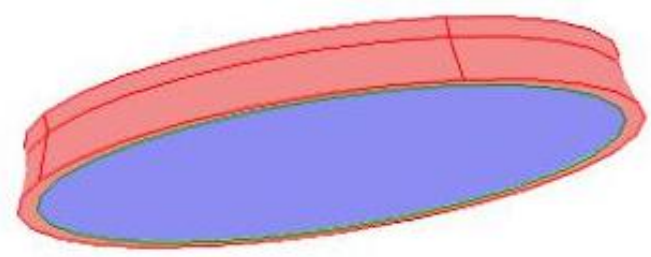

(a)

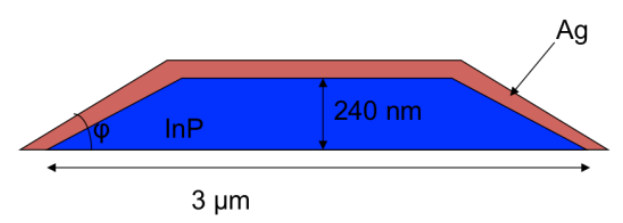

(b)

Figure(1) geomatry of cone-metal shelled structure, (a) 3D view of the geomatry each matrial is shown in different color InP layer in blue, coated by metallic silver cone. (b) side viewof the geomatry with structure dimensions.

\subsection{Study Mode profile}

In this work, it is focused on two kinds of modes, one is called hybrid plasmonic mode and second is pure plasmonic mode. In hybrid mode, the electric field concentrates on two positions, one on the dielectric region, which is also called dielectric mode, and the second on the interface between metal and dielectric. Figure 2(a,b) shows the side view and top view of the magnetic field distribution of the odd mode for hybrid plasmonic mode. As in the figure, part of the energy concentrated on the sidewall, and part of energy concentrate on dielectric region and this why it called hybrid mode.

In pure plasmonic mode the electric field only concentrates on the interface i.e. between metal and dielectric interface. Figure $2(c, d)$ shows the side view and top view of the magnetic field distribution of the even mode for pure plasmonic mode. As in the figure, the energy concentrated on the sidewall, i.e at the interface between metal and dielectric, for pure plasmonic mode. However, it is expected the Quality factor $(\mathrm{Q})$ is higher in hybrid mode than in pure plasmonic mode. The mode was a whispering-gallery (WG)-like SP polariton mode, and the electric field directions were perpendicular to the NR sidewall and the metal surface. 


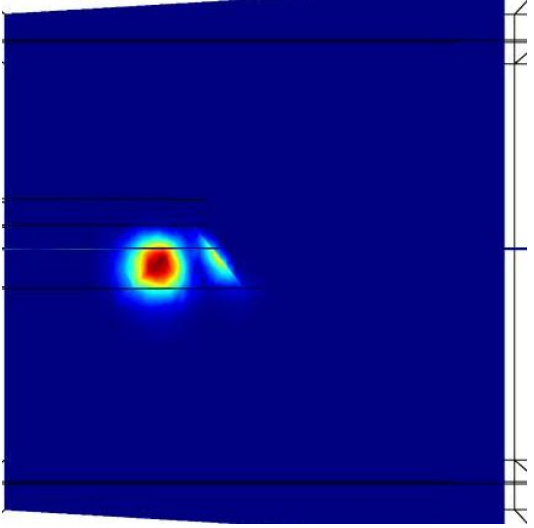

(a)

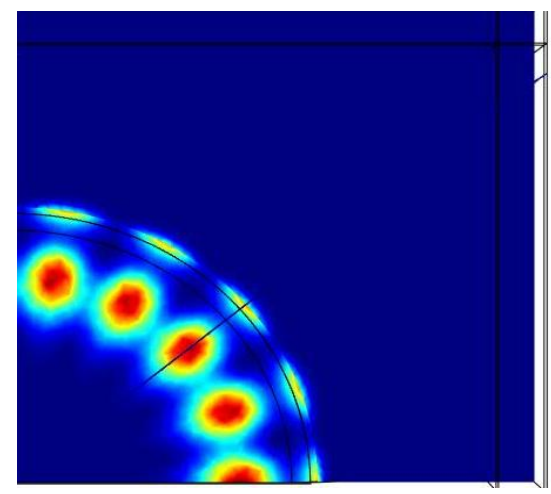

(b)

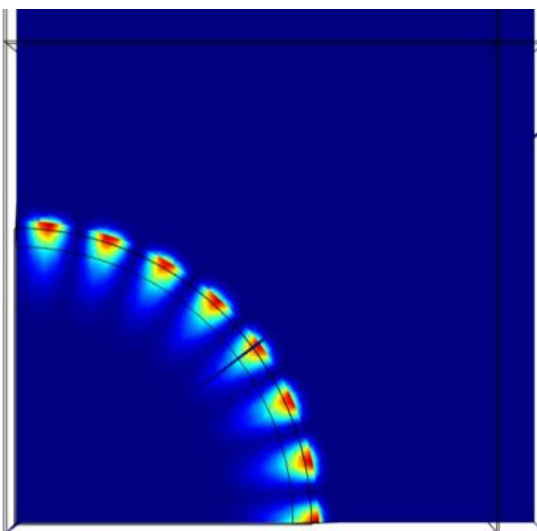

(c)

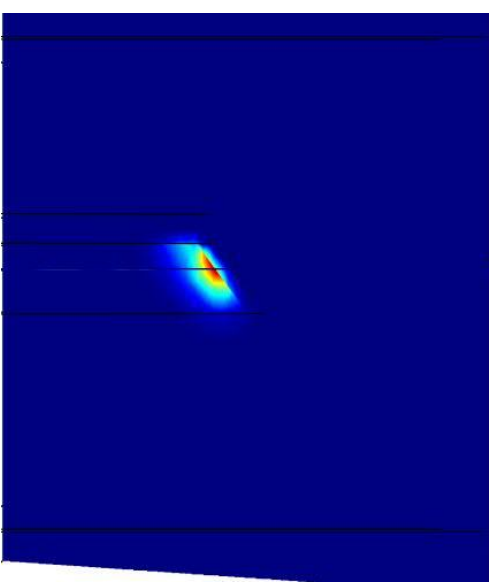

(d)
Figure (2): Field distribution of cone-metal shelled at resonance wavelength a) side view for hybrid plasmonic mode. b) top view for hybrid plasmonic mode. c) side view for pure plasmonic mode. d) side view for hybrid plasmonic mode.

\section{RESULT AND DISCUSSION}

The structure has been simulated with different sidewall angle $\Phi$ to examine the quality factor. Figure 3 shows Quality factor (Q) versus sidewall angle $\Phi$ for hybrid plasmonic modes. In the figure, the even mode and odd mode were examined; they show similar property for Quality factor. In both cases, it is found $\mathrm{Q}=775$ at sidewall angle ranged $\left(60^{\circ}-80^{\circ}\right)$ and the wavelength is $1.48 \mu \mathrm{m}$ for odd mode. Figure 4 shows Quality factor $(\mathrm{Q})$ versus sidewall angle $\Phi$ for pure plasmonic modes. In the figure, the even mode and odd mode were examined; they show similar property for Quality factor. In both cases, it is found $\mathrm{Q}=310$ at sidewall angle ranged $\left(60^{\circ}-70^{\circ}\right)$ but the wavelength is $1.5 \mu \mathrm{m}$ for even mode and $1.46 \mu \mathrm{m}$ for odd mode.

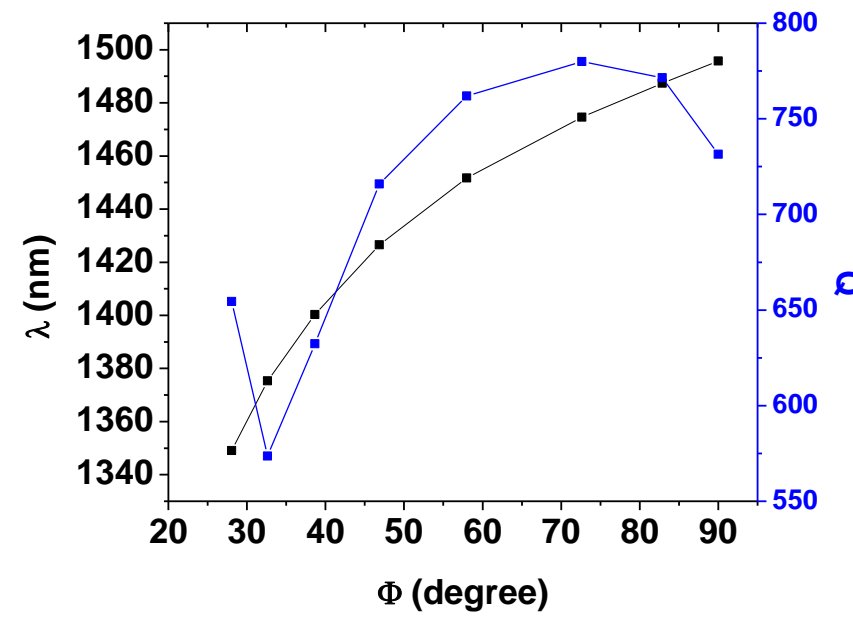

Figure 3 Quality factor $(Q)$ versus sidewall angle $\Phi$ for hybrid plasmonic modes shown in blue and resonance wavelengths $(\lambda)$ versus sidewall angle $\Phi$ for hybrid plasmonic modes shown in black.

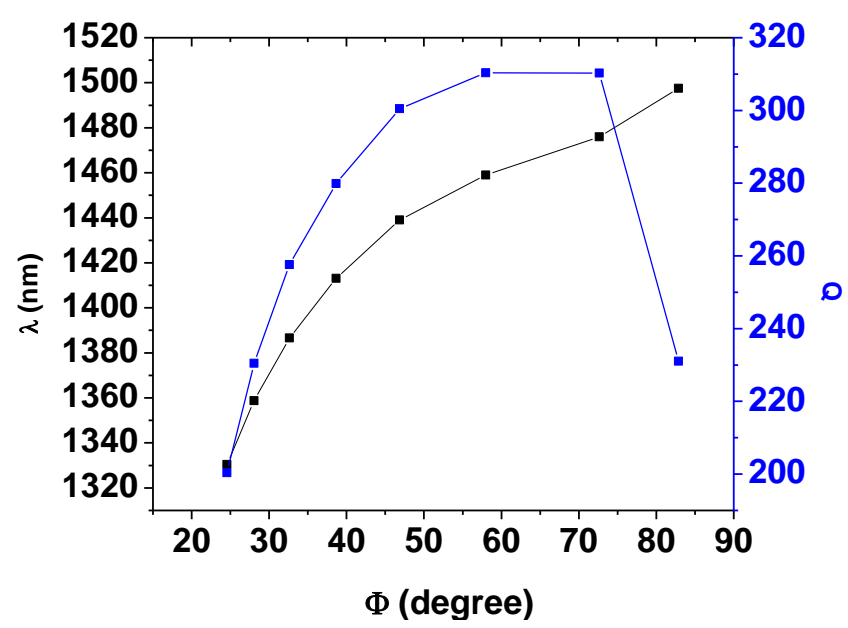

Figure 4 Quality factor $(Q)$ versus sidewall angle $\Phi$ for pure plasmonic modes shown in blue and resonance wavelengths $(\lambda)$ versus sidewall angle $\Phi$ for pure plasmonic modes shown in black. 
Thus, the quality factor in hybrid mode is higher than in pure plasmonic due to metallic loss. The main challenge associated with using plasmonic cavities is the loss that mainly comes from metal absorption in the visible and near-infrared wavelength range. Although some plasmonic modes, such as long-range surface plasmons, suffer less loss and propagate longer distances, the confinement of these modes becomes much worse. There is a fundamental compromise between loss and confinement for plasmonic modes. One of the causes that play an important role in the loss is electron collisions, which increase further with temperature. However, it is worth to know that the metallic loss depends on temperature. Here in this paper, it has been examined the temperature dependent in our simulation.

The metal-loss-limited Q factor depends predominantly on the damping collision frequency, $\gamma[6,14]$. To present a low temperature in our simulation, $\gamma$ was scaled by a factor of the room-temperature conductivity of a metal divided by the lowtemperature conductivity. Thus, we simulate our structure with different temperature values and examine the two obtained modes, i.e. the pure plasmonic modes and hybrid plasmonic mode. The quality factor of pure plasmonic mode increases slightly from room temperature degree $(300 \mathrm{~K})$ toward low temperature until reach $50 \mathrm{~K}$ afterward when the temperature decreases further towards $0 \mathrm{~K}$, the quality factor increases dramatically as shown in figure (5). The quality factor for pure plasmonic mode exceeds the $1.4 \mathrm{M}$. This result shows that the temperature play a very important role on plasmonic quality factor. However, as we mentioned before the pure plasmonic mode is in touch with metallic part of the structure therefore the increase in quality factor in pure plasmonic mode is due to low temperature and this causes low electron collision and eventually low loss.

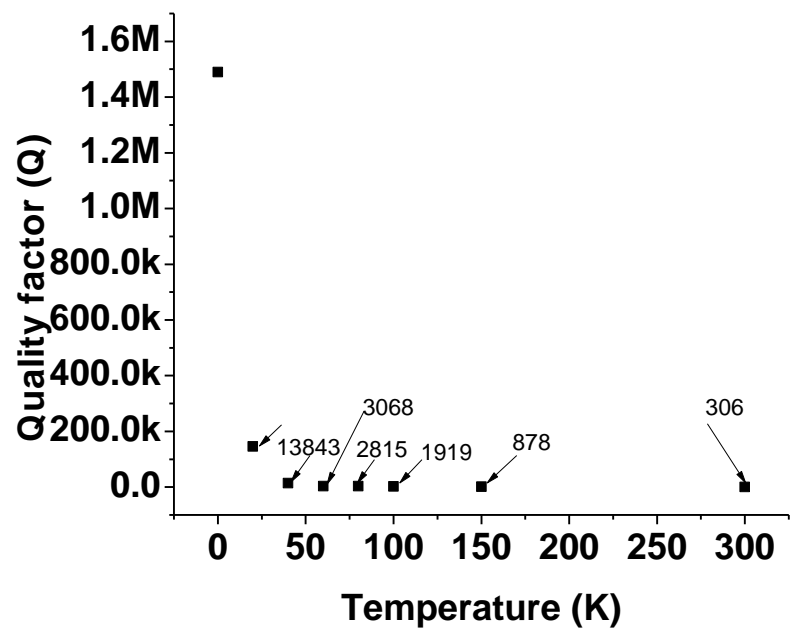

Figure (5): Quality factor (Q) versus Temperature (K) for pure plasmonic modes.

Also, we have examine the low temperature for hybrid plasmonic mode and the results show that also increase in the quality factor but with values much smaller as we have seen in pure plasmonic mode, see figure (6). In comparison, at $0 \mathrm{~K}$ temperature the pure plasmonic mode exceeds $1.4 \mathrm{M}$ whereas the hybrid plasmonic mode exceeds only 70,000. Therefore, we can say that the quality factor of plasmonic mode has inherently very large value (more than two order of magnitude) comparing with hybrid mode. In spite of the quality factor of hybrid mode is superior comparing with pure plasmonic mode at room temperature because of metallic loss but we expect the situation will be very different if we can decrease or remove the effect of metal loss at room temperature.

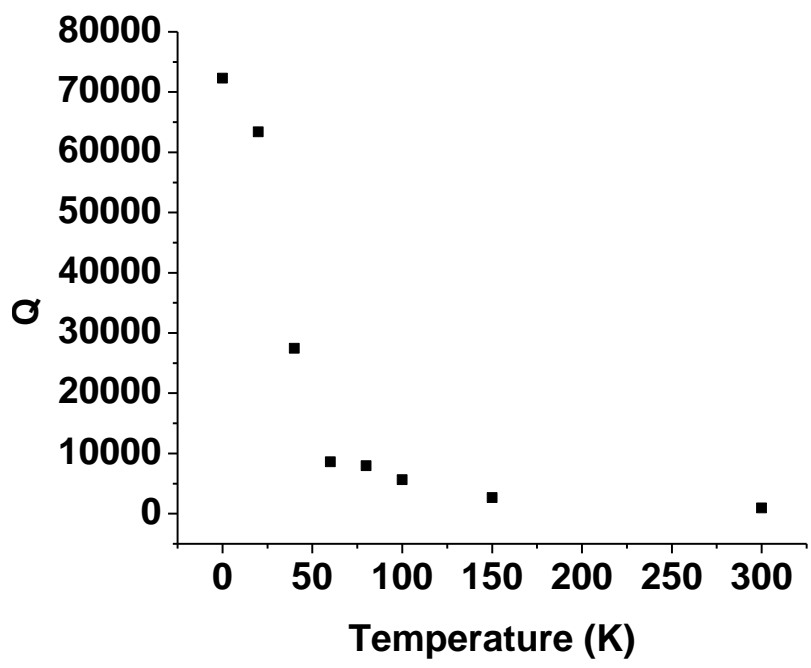

Figure (6): Quality factor $(Q)$ versus Temperature (K) for pure plasmonic modes.

\section{CONCLUSION}

In this paper, we have studied the quality factor the conemetal shelled structure. The two modes namely, hybrid and pure plasmonic mode, were studied and we found the quality factor for two modes can be maximized when the sidewall angle $\Phi$ is between $\left(60^{\circ}-70^{\circ}\right)$. For hybrid plasmonic mode the maximum quality factor is 775 at room temperature. For pure plasmonic mode the maximum quality factor is 310 at room temperature. Furthermore, we examined our structure at low temperature, at same sidewall angle the quality factor for pure plasmonic can exceed $1.4 \mathrm{M}$ at $0 \mathrm{~K}$ which is about 2 order of magnitude more than hybrid plasmonic mode at similar condition.

\section{REFERENCES}

[1] V. J. Sorger and X. Zhang," Spotlight on Plasmon Lasers" Science 333, 709 (2011).

[2] D. K. Gramotnev and S. I. Bozhevolnyi, "Plasmonics beyond the diffraction limit" Nat. Photonics 4, 83 (2010).

[3] M. T. Hill, Y.-S. Oei, B. Smalbrugge, Y. Zhu, T. de Vries, P. J. van Veldhoven, F. W. M. van Otten, T. J. Eijkemans, J. P. Turkiewicz, H. de Waardt, E. J. Geluk, S.-H. Kwon, Y.-H. Lee, R. Notzel, and M. K. Smit," Lasing in metallic-coated nanocavities" Nat. Photonics 1, 589 (2007).

[4] M. T. Hill, M. Marell, E. S. P. Leong, B. Smalbrugge, Y. Zhu, M. Sun, P. J. van Veldhoven, E. J. Geluk, F. Karouta, Y.-S. Oei, R. Notzel, C.-Z. Ning, and M. K. Smit, "Lasing in metal-insulator-metal sub-wavelength plasmonic waveguides "Opt. Express 17, 11107 (2009).

[5] K. Yu, A. Lakhani, and M. C. Wu, "Subwavelength metal-optic semiconductor nanopatch lasers" Opt. Express 18, 8790 (2010).

[6] Y.-G. Wang, C.-C. Chen, C.-H. Chiu, M.-Y. Kuo, M. H. Shih, and H.-C. Kuo, "Lasing in metal-coated $\mathrm{GaN}$ nanostripe at room temperature"Appl. Phys. Lett. 98, $131110(2011)$ 
[7] Weeber J-C, Lacroute Y, Dereux A, Devaux E, Ebbesen T, Girard C, Gonz'alez M U and Baudrion A-L, "Near field characterization of Bragg mirrors engraved in surface plasmon waveguides" Phys. Rev. B 70235406 (2004)

[8] Liu Z-W, Wei Q-H and Zhang X, "Surface plasmon interference nanolithography", Nano Lett. 5 957-61 (2005)

[9] Wang B and Wang G P Plasmon Bragg reflectors and nanocavities on flat metallic surfaces Appl. Phys. Lett. 87013107 (2005)

[10] Gong Y and Vuckovic J, "Design of plasmon cavities for solid-state cavity quantum electrodynamics applications", Appl. Phys. Lett. 90033113 (2007).

[11] Weeber J-C, Bouhelier A, des Francs G C, Markey L and Dereux A, "Submicrometer in-plane integrated surface plasmon cavities" Nano Lett. 7 1352-9 (2007).
[12] Kocabas A, Senlik S S and Aydinli A, "Plasmonic bandgap cavities on biharmonic gratings" Phys. Rev. B 77195130 (2008).

[13] Carrie E. Hofmann, Ernst Jan R. Vesseur, Luke A. Sweatlock, Henri J. Lezec, F. Javier García de Abajo ,Albert Polman , and Harry A. Atwater, "Plasmonic modes of annular nanoresonators imaged by spectrally resolved cathode luminescence" Nano Lett. 7 3612-7. (2007)

[14] Ditlbacher H, Hohenau A, Wagner D, Kreibig U, Rogers M, Hofer F, Aussenegg F R and Krenn J R Silver, "nanowires as surface plasmon resonators" Phys. Rev. Lett. 95257403 (2005)

[15] Miyazaki H T and Kurokawa Y, "Squeezing visible light waves into a 3-nm-thick and 55-nm-long plasmon cavity" Phys. Rev. Lett. 96097401 (2006)

[16] E.D. Palik, Handbook of Optical constants of solids (Academic press, 1998). 